PROCEEDINGS OF THE

AMERICAN MATHEMATICAL SOCIETY

Volume 126, Number 4, April 1998, Pages 1165-1172

S 0002-9939(98)04686-3

\title{
CONFORMAL FLATNESS AND SELF-DUALITY OF THURSTON-GEOMETRIES
}

\author{
STEPHAN MAIER
}

(Communicated by Ronald A. Fintushel)

\begin{abstract}
We show which Thurston-geometries in dimensions 3 and 4 admit invariant conformally flat or half-conformally flat metrics.
\end{abstract}

\section{ThrEe- AND FOUR-DIMENSIONAL GEOMETRIES}

A geometry in the sense of Thurston is a pair $(X, G)$ where $X$ is a simply connected smooth manifold on which the connected Lie group $G$ acts smoothly in a transitive fashion such that the following hold:

(1) the stabilizer of a point is compact;

(2) $G$ has a discrete subgroup $\Gamma$ such that $\Gamma \backslash X$ has finite volume for some $G$-invariant smooth measure;

(3) $G$ is maximal with these properties.

Maximality means that if there is a pair $\left(X^{\prime}, G^{\prime}\right)$ satisfying (1) and (2) together with a diffeomorphism $f: X \rightarrow X^{\prime}$ and an injective homomorphism of Lie groups $\phi: G \rightarrow G^{\prime}$ such that $f$ is $\phi$-equivariant, then $\phi$ must in fact be an isomorphism. We shall refer to $G$ as the structure group of the geometry, and $X$ will be called model space. Of course, such a manifold $X$ can be furnished with a Riemannian metric such that $G$ acts via isometries. But there is a whole range of possible choices provided the isotropy groups are small.

In dimension three, such geometries have been classified by Thurston (for an account see [Sco]), and in dimension four this has been done by Filipkiewicz [Fil], see also [Wall1]. The reader is refered to [Pat] for a general approach to the classification using Cartan triples. For later reference we shall collect here the possible different spaces $X$ which appear in these lists together with the identity component of the stabilizers of the action (see Table 1).

For simplicity we shall refer to the geometries $\widetilde{S L_{2}}(\mathbb{R}), N_{i l}^{3}, S o l^{3}, S o l_{0}^{4}, F^{4}, N i l^{4}$, $S_{0 l}^{4}{ }_{m, n}, S_{0} l_{1}^{4}$ as Lie group geometries. Observe that for any Lie group geometry $(X, G)$ the model-space $X$ is itself a Lie group, and the group $G$ contains $X$ as a subgroup. $F^{4}$ is the only geometry which does not admit compact models.

A manifold $M$ is said to have a geometry of type $(X, G)$ provided $M$ is covered by a collection of open sets each diffeomorphic to some open set in $X$ such that the transition functions are given by elements of $G$.

If the manifold $X$ in a pair $(X, G)$ admits a geometric structure such as a metric or an almost complex structure invariant under the action of $G$, this structure

Received by the editors July 23, 1996.

1991 Mathematics Subject Classification. Primary 53A35. 


\section{TABLE 1}

\begin{tabular}{|l|l|}
\hline Stabilizer & Geometry \\
\hline $\mathrm{SO}_{3}$ & $E^{3}, S^{3}, H^{3}$ \\
$\mathrm{SO}_{2}$ & $\mathrm{~S}^{2} \times E^{1}, H^{2} \times E^{1}, \widetilde{S L_{2}}(\mathbb{R}), N i l^{3}$ \\
$\{1\}$ & $\mathrm{Sol}^{3}$ \\
\hline $\mathrm{SO}_{4}$ & $\mathrm{~S}^{4}, \mathrm{E}^{4}, H^{4}$ \\
$\mathrm{U}_{2}$ & $\mathrm{CP}^{2}, \mathrm{CH}^{2}$ \\
$\mathrm{SO}_{2} \times S O_{2}$ & $\mathrm{~S}^{2} \times S^{2}, S^{2} \times E^{2}, \mathrm{~S}^{2} \times H^{2}, E^{2} \times H^{2}, H^{2} \times H^{2}$ \\
$\mathrm{SO}_{3}$ & $\mathrm{~S}^{3} \times E^{1}, H^{3} \times E^{1}$ \\
$\mathrm{SO}_{2}$ & $\widetilde{S L_{2}}(\mathbb{R}) \times E^{1}, \mathrm{Nil}^{3} \times E^{1}, \mathrm{Sol}_{0}^{4}, F^{4}$ \\
$\{1\}$ & $\mathrm{Nil}^{4}, \mathrm{Sol}_{m, n}^{4}, \mathrm{Sol}_{1}^{4}$ \\
\hline
\end{tabular}

descends to manifolds modelled on $(X, G)$. In the sequel such structures shall be labelled with the attribute 'invariant'. The case of complex structures has been extensively discussed in [Wall1], [Wall2], [K]. Here we shall be concerned with metrics which are either conformally flat or half-conformally flat. We obtain the following results:

Theorem 1. Apart from the flat geometry $E^{3}$ only the following geometries admit invariant conformally flat metrics: $H^{3}, S^{3}, S^{2} \times E^{1}, H^{2} \times E^{1}$.

Theorem 2. Apart from the flat geometry $E^{4}$ only the following geometries admit invariant conformally flat metrics: $S^{4}, H^{4}, S^{2} \times H^{2}, S^{3} \times E^{1}, H^{3} \times E^{1}$. Only the $C P^{2}$ - and $C^{2} H^{2}$ geometries admit invariant self-dual metrics which are not conformally flat. No geometry admits invariant non-conformally flat metrics which are anti-self-dual with respect to the standard orientation.

We finally address the question of the existence of 'non-geometric' conformally flat metrics on closed manifolds modelled on a Thurston geometry. It is known [Go] that closed 3-manifolds modelled on either $\mathrm{Nil}^{3}$ or $\mathrm{Sol}^{3}$ do not admit any conformally flat metric. We shall show that in dimension four a similar result holds (this result seems to be known to the experts but to our knowledge it has not appeared in print):

Theorem 3. Closed 4-manifolds modelled on either of $\mathrm{Nil}^{4}, \mathrm{Sol}_{m, n}^{4}$ or $\mathrm{Sol}_{1}^{4}$ do not admit any conformally flat metric.

This might be viewed as a global version of Theorem 2 . There we essentially discussed the existence of conformally flat metrics on local models, whereas Theorem 3 asserts that non-existence of conformally flat metrics results from the topology of the geometric manifold.

\section{Decomposition of the Curvature tensor}

The decomposition of the curvature tensor involves the Kulkarni-Nomizu product which is a linear map $S^{2}\left(S^{2} V\right) \rightarrow S^{2}\left(\Lambda^{2} V\right)$, where $V$ is any vector space. It is given by

$$
\begin{aligned}
h_{1} \bigotimes h_{2}(x, y, z, w)= & h_{1}(x, z) h_{2}(y, w)+h_{1}(y, w) h_{2}(x, z) \\
& -h_{1}(x, w) h_{2}(y, z)-h_{1}(y, z) h_{2}(x, w) .
\end{aligned}
$$


If $V$ is a real vector space of dimension $\operatorname{dim} V \geq 3$ furnished with a metric $g$, then the map

$$
\begin{aligned}
\phi_{g}: S^{2} V & \longrightarrow S^{2}\left(\Lambda^{2} V\right) \\
h & \longmapsto g \bowtie h
\end{aligned}
$$

is injective. Using this product the curvature tensor $R$ of a Riemannian manifold $(M, g)$ can be written as

$$
R=g \bowtie h+W
$$

Here

$$
\begin{aligned}
& \operatorname{dim} M=1: h=0, W=0, \\
& \operatorname{dim} M=2: h=\frac{s}{2} g, W=0, \\
& \operatorname{dim} M=3: h=\operatorname{Ric}-\frac{s}{4} g, W=0, \\
& \operatorname{dim} M \geq 4: h=\frac{1}{m-2}\left(\operatorname{Ric}-\frac{s}{2(m-1)} g\right),
\end{aligned}
$$

where $s$ is the scalar curvature function and $m=\operatorname{dim} M$. The tensor $W$, which is orthogonal to the image of $\phi_{g}$ in $S^{2}\left(\Lambda^{2} T^{*} M\right)$, is called the Weyl-tensor. The vanishing of the Weyl-tensor is the obstruction to conformal flatness, provided that $\operatorname{dim} M \geq 4$. In dimension three the condition for conformal flatness is the vanishing of the tensor

$$
d^{\nabla} h=A_{1,2}(\nabla h),
$$

where $A_{1,2}$ denotes antisymmetrisation in the first two slots [Laf2, p.74]. It is immediate that spaces of constant curvature are conformally flat. Of course, 1dimensional Riemannian manifolds are flat, and 2-dimensional Riemannian manifolds are conformally flat [Be, 1.169].

In dimension four $W$ further splits into its self-dual and anti-self-dual parts

$$
W=W^{+}+W^{-}, \quad W^{+} \in S_{0}^{2}\left(\Lambda^{+} T^{*} M\right), W^{-} \in S_{0}^{2}\left(\Lambda^{-} T^{*} M\right) .
$$

On a compact Riemannian 4-manifold there is the relation

$$
\sigma(M)=\frac{1}{12 \pi^{2}} \int_{M}\left(\left|W^{+}\right|^{2}-\left|W^{-}\right|^{2}\right) \operatorname{dvol}_{g}
$$

where $\sigma(M)$ denotes the signature of $M$.

In the sequel we shall make use of the following two propositions:

Lemma 1. If $(M, g)$ is a Riemannian product manifold

$$
(M, g)=\left(X_{1} \times X_{2}, g_{1}+g_{2}\right),
$$

then $(M, g)$ is conformally flat if and only if one of the following holds:

(1) One of $X_{1}, X_{2}$ is 1-dimensional and the other has constant curvature.

(2) Both $X_{1}, X_{2}$ have constant curvature of equal absolute value but opposite sign.

For a proof see for instance [Laf1, p.77].

Lemma 2. A product Riemannian 4-manifold $(M, g)=\left(X_{1} \times X_{2}, g_{1}+g_{2}\right)$ is (anti-)self-dual if and only if it is conformally flat. 
Proof. If $M$ is compact this is clear because the signature of $M$ is $\tau(M)=0$. In general, if, say, $\operatorname{dim} X_{1}=1, X_{1}$ has an orientation reversing isometry $\psi$ which induces the isometry $\psi \times I d$ on $M$, for which $(\psi \times I d)^{*} W^{+}=W^{-}$, and the result follows. If $\operatorname{dim} X_{1}=\operatorname{dim} X_{2}=2$,

$$
W_{M}=\frac{1}{12}\left(s_{1}+s_{2}\right)\left(g_{1} \oslash g_{1}+g_{2} \oslash g_{2}\right)-\frac{1}{6}\left(s_{1}+s_{2}\right) g_{1} \oslash g_{2}
$$

and the result follows by inspection of the formula.

\section{The proof of Theorems 1 And 2}

Proof. The geometries $E^{3}, S^{3}, H^{3}, S^{2} \times E^{1}, H^{2} \times E^{1}, S^{4}, E^{4}, H^{4}, S^{2} \times H^{2}$ have as structure group the isometry groups of the obvious Riemannian (product) metrics. By the results of the previous section these metrics are conformally flat.

The only metrics invariant under the structure groups of the geometries $S^{2} \times S^{2}$, $S^{2} \times E^{2}, H^{2} \times E^{2}, H^{2} \times H^{2}$ are up to rescaling in either factor the usual product metrics. The results of the previous section show that no such metric is conformally flat or (anti-)self-dual.

The structure groups of the geometries $C P^{2}$ and $C H^{2}$ are the isometry groups of the Fubini-Study metric and the Bergmann metric respectively. Both these metrics are self-dual but not conformally flat.

A metric on $N i l^{3} \times E^{1}$ is invariant if and only if its restriction to $N i l^{3} \times\{p\}$ for some $p$ is invariant and the metric is translationally invariant. A similar remark applies to $\widetilde{S L_{2}}(\mathbb{R}) \times E^{1}$. It is thus sufficient to consider Lie group geometries only to conclude the proof.

By Theorem 1 of $[\mathrm{M}]$, a simply connected Lie group of dimension $n \geq 3$ which carries a left invariant conformally flat metric must be isometric (up to rescaling) to either $E^{n}, H^{n}, S^{3}$, or the Riemannian products $E^{1} \times S^{3}, E^{1} \times H^{n-1}, S^{3} \times H^{n-3}$ with the standard metrics. Note that for any Lie group geometry $(X, G), G$ contains the Lie group $X$ as a subgroup, and thus a metric invariant under the structure group must be a left invariant metric on $X$. If a Lie group geometry did admit an invariant conformally flat metric, this would identify the Lie group geometry as one of $E^{n}, H^{n}, S^{3}, E^{1} \times S^{3}, E^{1} \times H^{n-1}, S^{3} \times H^{n-3}$, a contradiction. Alternatively, one could of course check that none of the Lie groups appearing in the list of Lie group geometries is isomorphic to a Lie group admitting a conformally flat left invariant metric.

In dimension four all geometries admit compact models apart from $F^{4}$. Thus no Lie group geometry with the possible exception of the geometry $F^{4}$ admits invariant (anti-)self-dual metrics; for by [Wall1, Th.6.1] the signature of compact models is $\sigma=0$. Thus $W^{+}=0$ implies $W^{-}=0$ and vice versa by equation (1) above, and hence an (anti-)self-dual metric would necessarily be conformally flat.

There remains the case $F^{4}$ : The model space $X$ for this geometry is $\mathbb{R}^{2} \times H^{2}$, where $H^{2}$ stands for the upper half-plane. The structure group $G$ is $\mathbb{R}^{2} \rtimes S l_{2}(\mathbb{R})$ with the natural structure of semidirect product. This $G$ acts on $X$ by the rule

$$
\left(\left(\begin{array}{l}
u \\
v
\end{array}\right),\left(\begin{array}{ll}
a & b \\
c & d
\end{array}\right)\right) \cdot\left(\left(\begin{array}{l}
x \\
y
\end{array}\right), z\right):=\left(\left(\begin{array}{l}
u+a x+b y \\
v+c x+d y
\end{array}\right), \frac{a z+b}{c z+d}\right) .
$$

The stabilizer of the point $(\overrightarrow{0}, i)$ is $S O_{2} \subset S l_{2}(\mathbb{R})$, and $X$ is identified with $F^{4}=$ $R^{2} \rtimes A$ via the map $\pi: f \mapsto f \cdot(\overrightarrow{0}, i)$, where $A \subset S l_{2}(\mathbb{R})$ are the upper triangular 
matrices. See [Wall1, p.122]. The vectors

$$
\begin{aligned}
& e_{1}=\left(\left(\begin{array}{l}
1 \\
0
\end{array}\right),\left(\begin{array}{ll}
0 & 0 \\
0 & 0
\end{array}\right)\right), e_{2}=\left(\left(\begin{array}{l}
0 \\
1
\end{array}\right),\left(\begin{array}{ll}
0 & 0 \\
0 & 0
\end{array}\right)\right), \\
& e_{3}=\left(\left(\begin{array}{l}
0 \\
0
\end{array}\right),\left(\begin{array}{cc}
1 & 0 \\
0 & -1
\end{array}\right)\right), e_{4}=\left(\left(\begin{array}{l}
0 \\
0
\end{array}\right),\left(\begin{array}{ll}
0 & 2 \\
0 & 0
\end{array}\right)\right)
\end{aligned}
$$

span the Lie algebra of $F^{4}$. One computes the following brackets:

$$
\left[e_{3}, e_{1}\right]=e_{1},\left[e_{3}, e_{2}\right]=-e_{2},\left[e_{3}, e_{4}\right]=2 e_{4},\left[e_{4}, e_{2}\right]=2 e_{1}
$$

and the other brackets all vanish. Note that the differential $\pi_{*}$ of $\pi$ at the identity maps the vectors $\left\{e_{1}, e_{2}\right\}$ to the standard basis of $\mathbb{R}^{2}$, and it maps $e_{3}$ and $e_{4}$ to the vectors $2 i$ and 2 in the complex plane respectively. Thus the stabilizer $\mathrm{SO}_{2}$ acts isometrically on the tangent space at $(\overrightarrow{0}, i) \in X$ if we choose the metric for which $\left\{\pi_{*} e_{1}, \ldots, \pi_{*} e_{4}\right\}$ is an ON-basis. It is thus clear that the metric induced by the frames $\left\{\pi_{*} E_{1}, \ldots, \pi_{*} E_{4}\right\}$ is invariant under the action of $G$, where $E_{i}$ is the left-invariant vector field induced on $F^{4}$ by $e_{i}$. Note that this metric is not the standard metric on $\mathbb{R}^{2} \times H^{2}$.

It is a straightforward albeit tedious exercise to compute the Weyl tensor for this metric. The recipe is to first compute the covariant derivative using the identity

$$
2\left\langle\nabla_{e_{i}} e_{j}, e_{k}\right\rangle=-\left\langle e_{i},\left[e_{j}, e_{k}\right]\right\rangle+\left\langle e_{k},\left[e_{i}, e_{j}\right]\right\rangle+\left\langle e_{j},\left[e_{k}, e_{i}\right]\right\rangle
$$

Then the curvature tensor is computed using

$$
R\left(e_{i}, e_{j}, e_{k}, e_{l}\right)=\left\langle\nabla_{\left[e_{i}, e_{j}\right]} e_{k}, e_{l}\right\rangle+\left\langle\nabla_{e_{i}} e_{l}, \nabla_{e_{j}} e_{k}\right\rangle-\left\langle\nabla_{e_{i}} e_{k}, \nabla_{e_{j}} e_{l}\right\rangle .
$$

Hence the Ricci curvature is $\operatorname{Ric}\left(e_{i}, e_{j}\right)=\sum_{k} R\left(e_{i}, e_{k}, e_{j}, e_{k}\right)$, and the scalar curvature is $s=\operatorname{Tr}(\operatorname{Ric})=\sum_{k} \operatorname{Ric}\left(e_{k}, e_{k}\right)$. Eventually, the Weyl tensor is found using

$$
\begin{aligned}
W\left(e_{i}, e_{j}, e_{k}, e_{l}\right) & =R\left(e_{i}, e_{j}, e_{k}, e_{l}\right)+\frac{s}{12}\left(\delta_{i k} \delta_{j l}-\delta_{i l} \delta_{j k}\right) \\
- & \frac{1}{2}\left(\operatorname{Ric}\left(e_{i}, e_{k}\right) \delta_{j l}+\operatorname{Ric}\left(e_{j}, e_{l}\right) \delta_{i k}-\operatorname{Ric}\left(e_{i}, e_{l}\right) \delta_{j k}-\operatorname{Ric}\left(e_{j}, e_{k}\right) \delta_{i l}\right) .
\end{aligned}
$$

With a little patience one may now compute the Weyl tensor for $F^{4}$ :

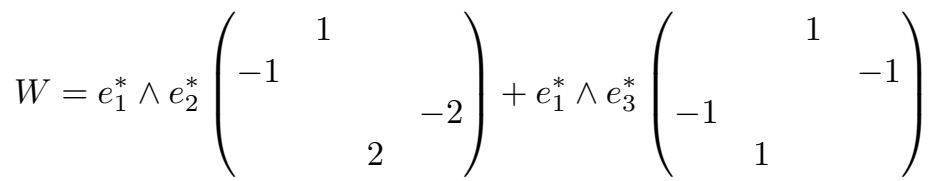

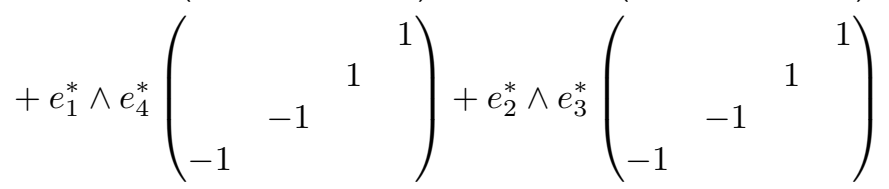

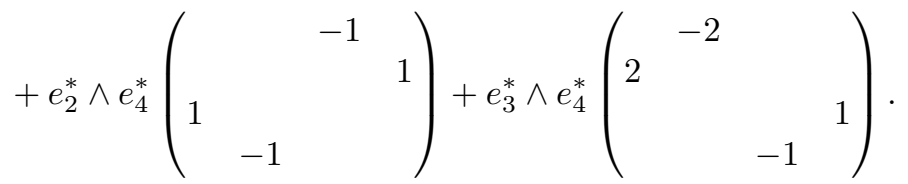

This tensor is manifestly not (anti-)self-dual, for example the coefficients of $e_{1}^{*} \wedge e_{2}^{*}$ and $e_{3}^{*} \wedge e_{4}^{*}$ do not differ by multiplication with \pm 1 only. 
The action of the stabilizer $\mathrm{SO}_{2}$ on the Lie algebra of $F^{4}$ is as follows: the action leaves invariant the spaces spanned by $e_{1}, e_{2}$ (where it acts isometrically with weight 1 ) and $e_{3}, e_{4}$ (where it acts isometrically with weight -2 ) respectively. It is thus clear that any invariant metric of $F^{4}$ (up to rescaling) has as ON-basis the following:

$$
e_{1}^{\prime}=\lambda e_{1}, \quad e_{2}^{\prime}=\lambda e_{2}, \quad e_{3}^{\prime}=e_{3}, \quad e_{4}^{\prime}=e_{4}, \quad \lambda \in \mathbb{R}^{+} .
$$

But the brackets of $\left\{e_{1}^{\prime}, \ldots, e_{4}^{\prime}\right\}$ are precisely the same as those of $\left\{e_{1}, \ldots, e_{4}\right\}$, and hence the Weyl tensor for the new metric is obtained from the above tensor $W$ by adding primes to every $e_{i}$. Thus no invariant metric of $F^{4}$ is (anti-)self-dual.

\section{The Proof of Theorem 3}

Proof. By possibly passing to a finite covering we may assume that a given manifold $M$ modelled on any of the three geometries $\mathrm{Nil}^{4}, \mathrm{Sol}_{m, n}^{4}$ or $\mathrm{Sol}_{1}^{4}$ is a quotient of the respective Lie group by a uniform discrete subgroup $\Gamma$. This is true because the stabilizers of these geometries are discrete. To see this let $(H, G)$ be one of these three geometries. Here, $H$ is the model-space and $G$ is the group implied in the geometry. Furnishing $H$ with a $G$-invariant metric, this metric induces a metric on $M$. The development map of the Riemannian universal cover $\tilde{M}$ into $H$ is a local isometry and by completeness of the metrics on $\tilde{M}$ and $H$ a covering map. By simple-connectivity of $H$ the development map is thus an isometry, and $M$ is a quotient of $H$ by a subgroup of $G$ (which we shall denote by $\pi_{1}(M)$ ). But $G / H$ is finite, and thus $\Gamma:=\pi_{1}(M) \cap H \subset \pi_{1}(M)$ is a subgroup of finite index. Thus the quotient $M^{\prime}:=G / \Gamma$ is a finite cover of $M$ and is a quotient of $H$ by a discrete uniform subgroup $\Gamma \subset H$.

Now suppose that $M$ admits a conformally flat metric. By Corollary 3.5 and Theorem 3.6 of [Kam], the universal cover of $M$ is conformally equivalent to either $S^{4}, \mathbb{R}^{1} \times S^{3}$ or $\mathbb{R}^{4}$ with their standard metrics. In the latter case $M$ is finitely covered by a torus. But by the description below, all three of $\mathrm{Nil}^{4}, \mathrm{Sol}_{m, n}^{4}$ and $S o l_{1}^{4}$ are isomorphic as manifolds to $\mathbb{R}^{4}$. Thus by possibly passing to a finite index subgroup we may assume that $\Gamma$ is isomorphic to $\mathbb{Z}^{4}$. Now consider the three geometries in turn.

The underlying space of $N i l^{4}$ is the semidirect product $\mathbb{R}^{3} \rtimes_{\alpha} \mathbb{R}$, where

$$
\alpha(t)=\exp \left\{t\left(\begin{array}{lll}
0 & 1 & 0 \\
0 & 0 & 1 \\
0 & 0 & 0
\end{array}\right)\right\} .
$$

By [Ra, 2.3], $\Gamma$ is Zariski-dense in the Lie group $N i l^{4}$. Hence $[\Gamma, \Gamma]$ is Zariski-dense in $\left[N i l^{4}, N i l^{4}\right]$. But by assumption, $[\Gamma, \Gamma]$ is trivial whereas $\left[N i l^{4}, N i l^{4}\right]$ has real dimension two, a contradiction.

The model space of the geometry $\operatorname{Sol}_{m, n}^{4}$ is the semidirect product $\mathbb{R}^{3} \rtimes_{\alpha_{m, n}} \mathbb{R}$, where

$$
\alpha_{m, n}(t)=\exp \left\{t\left(\begin{array}{lll}
a & 0 & 0 \\
0 & b & 0 \\
0 & 0 & c
\end{array}\right)\right\},
$$

where $a>b>c$ and $e^{a}, e^{b}$ and $e^{c}$ are the roots of $\lambda^{3}-m \lambda^{2}+n \lambda=1, m, n \in \mathbb{N}$ [Wall1, p.121]. The Nil-radical $N \subset S_{\text {Sol }}^{4}, n$ is euclidean of dimension three. By [Ra, 3.3] $\Gamma \cap N$ is discrete and uniform in $N$. Let $\gamma \in \Gamma \backslash \Gamma \cap N$. By assumption the 
action of $\gamma$ on $\Gamma \cap N$ by conjugation is trivial. But writing $\gamma=(x, \alpha(t))$ this action on $N$ is the linear action of $\alpha(t)$ on the vector space $N$, and because $\Gamma \cap N$ contains a base of $N$ this linear action is trivial. It follows that $\gamma \in N$, a contradiction.

Finally, consider $S_{0} l_{1}^{4}$. Its underlying space is the Lie group

$$
\left\{\left(\begin{array}{ccc}
1 & b & c \\
0 & \alpha & a \\
0 & 0 & 1
\end{array}\right), \alpha, a, b, c \in \mathbb{R}, \alpha>0\right\} .
$$

Here the Nil-radical is $N=N i l^{3} \subset S o l_{1}^{4}$. Here, $\Gamma \cap N$ is a discrete uniform subgroup $[\mathrm{Ra}, 3.3]$ and it is abelian by assumption. Thus $[\Gamma \cap N, \Gamma \cap N]$ is trivial, but by $[\mathrm{Ra}, 2.3]$ it must be Zariski-dense in $[N, N]$ which has real dimension one, again a contradiction.

Remark. The proof of this non-existence result is really quite general in nature. Suppose given a simply connected Lie group $G$ and a subgroup $G^{\prime}$ of the diffeomorphism group of $G$ such that $G \subset G^{\prime}$ in a natural way. Suppose that the stabilizer of the identity in $G$ is finite and equal to $G^{\prime} / G$. Suppose we are given a closed manifold with an atlas of charts each diffeomorphic to an open subset of $G$ such that the transition functions are given by restriction of elements of $G^{\prime}$. Suppose furthermore that the fundamental group of $M$ is solvable. Assuming $M$ did admit a conformally flat metric we obtain restrictions on the universal cover of $M$. If for instance $G$ is contractible, the fundamental group $\pi_{1}(M)$ of $M$ is a finite extension of $\mathbb{Z}^{\operatorname{dim} M}$. Now by possibly passing to a finite index subgroup of $\pi_{1}(M)$ we may assume that $M$ is actually a quotient of $G$ by a uniform discrete subgroup $\Gamma$. We may now apply the various theorems about discrete subgroups in Lie groups to perhaps derive a contradiction, thus showing that $M$ does not carry any conformally flat metric, or to draw conclusions about the structure of $M$ provided the existence of a conformally flat metric is known.

\section{ACKNOWLEDGEMENTS}

I wish to thank D. Kotschick for bringing to my attention the problems discussed here.

\section{REFERENCES}

[Be] A.L. Besse, Einstein Manifolds, Ergebnisse 3. Folge Band 10, Springer, 1987. MR 88f:53087

[Fil] R.O.Filipkiewicz, Four dimensional geometries, Ph.D. thesis, University of Warwick (1983).

[Go] W.Goldman, Conformally flat manifolds with nilpotent holonomy and the uniformization problem for 3-manifolds, Trans. Am. Math. Soc. 278 Nr. 2 (1983), 573-583. MR 84i: 53043

[K] D.Kotschick, Remarks on geometric structures on compact complex surfaces, Topology 31 (1992), 317-321. MR 93g:32038

[Kam] Y.Kamishima, Conformally flat manifolds, Trans. Am. Math. Soc. 294 Nr. 2 (1986), 607-623. MR 87g:57060

[KuPi] R.Kulkarni, U.Pinkall (eds.), Conformal Geometry, Aspects of Mathematics, Vieweg, 1988. MR 89g:53002

[Laf1] J.Lafontaine, Remarques sur les variétés conforméments plats, Math. Ann. 239 (1982), 313-319.

[Laf2] J.Lafontaine, Conformal geometry from the Riemannian viewpoint, in [KuPi]. MR 89g:53002

[M] S.Maier, Conformally flat Lie groups, Math. Zeitschrift, to appear. 
[Pat] V.Patrangenaru, Classifying 3 and 4 dimensional homogeneous Riemannian manifolds by Cartan triples, Pacific Jour. of Math. 173 No. 2 (1996), 511-532. MR 97e:53096

[Ra] M.S.Raghunathan, Discrete subgroups of Lie groups, Ergebnisse Band 68, Springer. MR 58:22394a

[Sco] S.Scott, The Geometries of 3-manifolds, Bull. London Math. Soc. 15 (1984), 401-487.

[Wall1] C.T.C.Wall, Geometric structures on compact complex surfaces, Topology 25 (1986), 119-153. MR 88d:32038

[Wall2] C.T.C.Wall, Geometries and geometric structures in real dimension four and complex dimension two, Lecture Notes in Math. 1167, Proceedings of the Univ. of Maryland, pp. 268-292. MR 87e:57023

Institut für Mathematik, Universität Zürich, Winterthurerstr. 190, 8057 Zürich, SWITZERLAND

E-mail address: stmaier@math.unizh.ch 